\title{
Benefit of Selecting Number of Active Mesh Routers in Disaster Oriented Wireless Mesh Network
}

\author{
Panu Avakul $^{1}$, Hiroki Nishiyama ${ }^{1}$, Nei Kato ${ }^{1}$, Yoshitaka Shimizu' ${ }^{2}$, Tomoaki Kumagai ${ }^{2}$ \\ ${ }^{1}$ Graduate School of Information Sciences, Tohoku University, Sendai, Japan; ${ }^{2}$ NTT Network Innovation Laboratories, NTT Corpo- \\ ration, Yokosuka, Japan. \\ Email: panu0014@it.ecei.tohoku.ac.jp, bigtree@it.ecei.tohoku.ac.jp, kato@it.ecei.tohoku.ac.jp,shimizu.yoshitaka@lab.ntt.co.jp, \\ kumagai.tomoaki@lab.ntt.co.jp
}

Received 2012

\begin{abstract}
Communications is one of the most critical factors in disaster recovery process. However, after a major disaster, existing communications infrastructures may be heavily damaged or even completely unusable. It is necessary that communicationsare to be promptly restored to the disaster area, which is the goal of our national project. The project aims to build three tiers wireless mesh network from remaining wireless access points in order to provide communications services to the disaster area. This work introduces a unique multiple tiers wireless mesh network project. In addition, this work also illustrates merits in optimizing the number of mesh routersin order to achieve the optimum performance by presenting both theoretical and simulation results of a specific scenario of multiple tiers wireless mesh network.
\end{abstract}

Keywords: Wireless Mesh Network; Throughput; Interference; Collision; WiFi

\section{Introduction}

Following the 2011 Tohoku earthquake and tsunami, the field of emergency network deployment has gainedmuch attention from research institutions all over Japan. This is because drastic disaster such as earthquake or tsunamiwill incapacitate the existing communications infrastructure. Therefore, it is important to be able to restore communications in the disaster area using whatever means necessary. In addition, it ismentioned by [1] that it is more complicated to deploy a new communications system where the previous system still exists. An ongoing national project aims to restore communications within the disaster area using the remaining resources, namely the remaining wireless access points, to constructs a wireless mesh backbone and to provide communications to users in the affected area who are referred to as Mesh Client (MC).

Wireless Mesh Network (WMN) is a type of infrastructure where each participating node or a Mesh Router (MR) both sends and relays information similar to an ad-hoc network with the exception that MR does not usually have power constraint or mobility problem. In addition, many MRs are also likely to act as wireless Access Point (AP) and provide communications service to MCs within its area similar to that of an ordinary wireless AP. WMN has been gaining attention from research communities due to the low-cost and rapid de- ployment, whichare suitable properties for an emergency network role. In addition, it also inherits many other valuable characteristics from ad-hoc network such decentralized design and distributed communications[2].

In addition to being able to relay information between MRs, MR is also usually equipped with an addition radio interface to acts as a wireless AP andto provide communications services to users within its area. This implies that the MCs will be able to connect to a network like connecting to WiFi hot spot, but the communication is relayed through the wireless mesh backbone instead of the traditional wired connection.Due to this unique characteristic of this multiple tiers WMN, the number of active MRs plays a significant role in determining the performance of the whole network.

It was mentioned that our project relies extensively on the remaining wireless APs that are already deployed in an uncontrolled manner,and number of active MRs can affect the performance in multiple-tiers of the project.Therefore, in order to deploy the project successfully with the optimum performance, we need to optimize the number of MRs. This work briefly introduces the national project and investigates the effect of using the optimum number of MRs in a unique WMN project. We introduce a theoretical performance of a specific scenario of multiple-tier WMN, which is backed up by simulation results.

The remaining sections of this paper are divided into following: Section 2introduces related works and dis- 
cusses the structure of the project. Section 3shows theoretical discussion of the scenario. Section 4 presents the simulation results and discussion. Finally, Section 5 presents the conclusion.

\section{Background}

\subsection{Related Works}

Reference [3]presents Extreme Networking System (ENS) architechture, which is a three tiers network that is very similar to our project's architechture. According to the article, ENS was experimentally deployed on November 2005, at San Diego and many useful emergency response statistics, such as traffic statistic, andperformance of the architechture, was collected. The authors also present many challenges in deploying wireless mesh network in the case of emergency response. One of the major difference of ENS and our project is that our project plans to rely on existing inflastruture such as remaining wireless APs. A more detail explaination regarding our project will be given in Section 2.2.

In addition to ENS presented by [3], authors of [4] also present another similar network architechture to our project called the hybrid WMN. Hybrid WMN aims to enhances the performance of metapolian WMN with the addition of wired APs. While hybrid WMN is not intended for usage in an emergency response situation, it is a great idea which may also help increase the overall performance of emergency response WMN. Since in an ideal situation, some remaining wireless APs may still have wired connection available.

Reference[5] investigates the impact of inter-cell interference on WLAN performance. The authors' testbed experiment shows that inter-cell inteference can greatly reduce the WLAN performance under the TCP dominated common office traffic pattern.

Reference [6] presents a novel gateway selection method for multiple tiers WMN that focuses on deployment in disaster area. The method presented ultilize the concept of Collision Domain (CD) to choose the most suitable MR to act as Mesh Gateway (MG)in order to have the best overall system throughput.

Reference [7] presents a deployment evaluation of the Roofnet wireless mesh network, which is an unplanned WMN. The author suggests that having a denser MR may help improve the average throughput of the network due to the posibility of chosing shorter high quality links with the cost of having a higher average hop-count. However, the author also shows that the performance suffers from multi-hops transmission due to inter-hop interference. In addtition, [8] shows that in a multi-hop WMN there is fairness problem where MRs that has a higher hop-count to the gateway achieve much lower throughput than those with lower hop-count.

\subsection{Project Structure and Characteristics}

\subsubsection{Three Tiers Network}

As shown in Figure 1, the project is composed of several components. The Moveable and Deployable Resource Units (MDRU) acts as a gateway that provides connections from the system to outside network such as internet. The MR is an ordinary wireless AP which is configured to act as wireless mesh backbone in order to provide communications service to the MCs. MG is a specific MR that communicates directly with the MDRU. The overall project can be partitions into three separated tiers: network facility tier, mesh tier, and clients tier.

The network facility tier covers the communication between the MDRU(s) and MGs, which is done in a special $25 \mathrm{GHz}$ band. The mesh tier is the wireless mesh backbone level where the MGs and MRs form a wireless mesh backbone network using the $5 \mathrm{GHz}$ band. The MGs act as mesh portal to bridge the communication between the network facility tier and the mesh tier. Finally, the clients tier is the level, which each MG and MR provide communications service to MCs within its vicinity over the $2.4 \mathrm{GHz}$ band. Since the links at the network facility tier are assumed to be high performance links, this work focus on the performance in mesh tier and clients tier.

\subsubsection{Unique Deployment}

A scenario of how the project will be deployed is the MDRU will be deploy to the disaster area prior to disaster. The MDRUis capable of carrying a certain number of MGs in order to have some control over the topology of the system. After arrival, the MDRU will configure any remaining wireless APs in the area into MRs or MGs and restore communications service to MCs in the area.

\subsubsection{Uncontrolled MRs Placement}

Another major characteristic of the project is that since MRs are just commonly deployed wireless APs, and it is pointed out by [9] that common 802.11 APs are usually deployed in an unplanned or random fashion. Therefore, there is a good chance that the deployment will not be optimized, and thus causing the drop in performance.

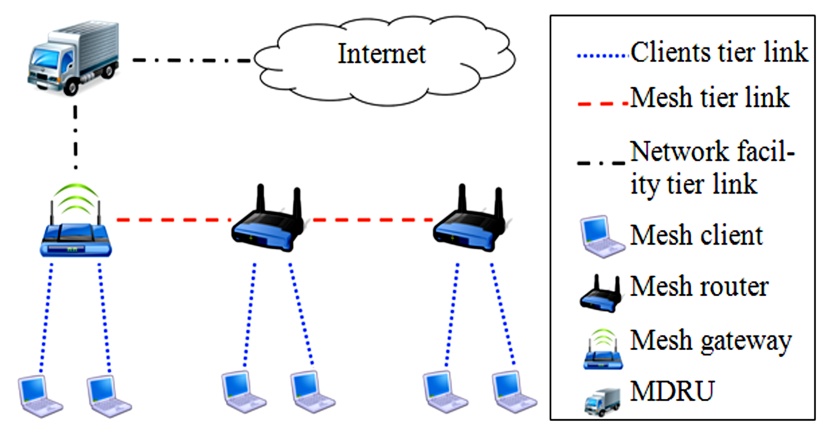

Figure 1.The structure of the project. 


\section{Effect of Number of MRs}

\subsection{Effect of Number of MRs}

$\mathrm{CD}$ is an important concept that can be used to estimate the capacity of WMN as shown in [10]. Since wireless links share the same medium, it is necessary that only one device within the same interfering range should be transmitting at any given time to have a successful transmission. In another word, if a certain link $n$ is active, any other link within the same interfering range of link $n$ must be inactive in order for transmission at link $n$ to be successful. A CD is defined by [10] to be a set of links that have to be inactive for a transmission at a certain link to be successful including the transmitting link itself. Figure 2 illustrates a chain topology of a two tiers WMN where there are six MRs, which can only transmit to an adjacent MR. The rightmost device is the MG that is assumed to be the sink of all traffic. Each MR has to transmit $G$ amount of traffic from its own clients tier and any other amount of traffic forwarded by its upstream MR(s). For example, MR2 has to forward $G$ amount of traffic from its clients tier plus what MR1 forwarded which is another $G$ to the total of $2 G$ amount of traffic. It is assumed that the interfering range is two times the transmission range; therefore, the CD of link between MR4 and MR5 is a set of $\{(M R 2, M R 3),(M R 3$, MR4),(MR4, MR5),(MR5, MR6),(MR6, MG)\}.

The bottleneck collision domain (BCD) is defined by [10] to be the CD that has to forward the most traffic. For instance, the CD of link MR4 and MR5 has to forward all traffic forwarded by each link within the set of its CD. Therefore, CD of link MR4 and MR5 has to forward total of $2 G+3 G+4 G+5 G+6 G=20 G$. The same calculation can also be done on $\mathrm{CD}$ of other links, but in the case of scenario in Figure 2,CD of link MR4 and MR5 has to forward the most traffic. Hence, it is the BCD of this chain topology. According to the previous calculation, $\mathrm{CD}$ of link between MR4 and MR5 has to forward 20G amount of traffic. However, the capacity is bound by the MAC layer capacity $C$, thus can be represent by

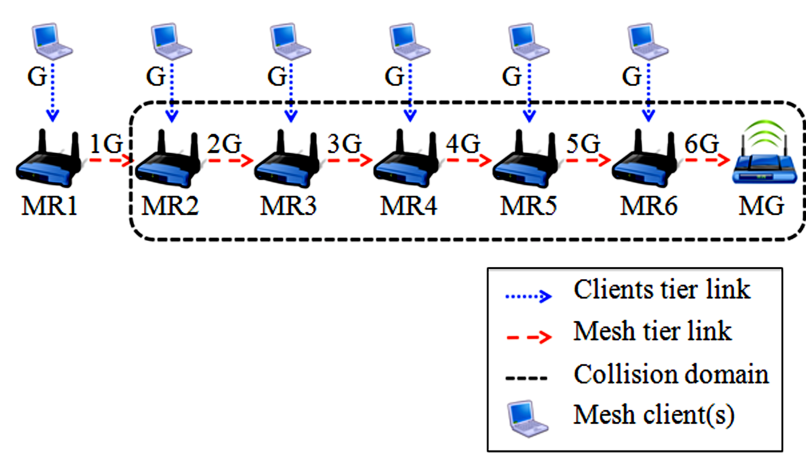

Figure 2. A chain topology where each MR sends the same amount of traffic to the MG.

$$
20 G \leq C .
$$

By solving inequality in Equation (1), we can see that the maximum throughput available to each $\mathrm{MR}$ or $G_{\max }$ is

$$
G_{\max }=\frac{C}{20}
$$

From the analysis above, we can see that the more general case of Equation (2) is

$$
G_{\max }=\frac{C}{A M T}
$$

where $A M T$ is the total amount of traffic forwarded by BCD divided by $G$, which will be 20 in the case of $C D$ of link MR4 and MR5.

\subsection{Considered Scenario}

In order to show the effect of number of MRs, we consider following scenario illustrates in Figure 3(a) where there are one MG, three MRs and six MCs. Some properties of the scenario are as following:

- All MCs can connect to any available MRs.(All MCs are within the communication range of any MRs.)

- Each MR operates in different channel in a way that they do not have inter-cell interference introduced in [5].

- The mesh tier links operate in one common channel.

- Each MRs will always have the same number of MCs within its cell.

In addition, this scenario can be narrow down to three sub-scenarios as follow:

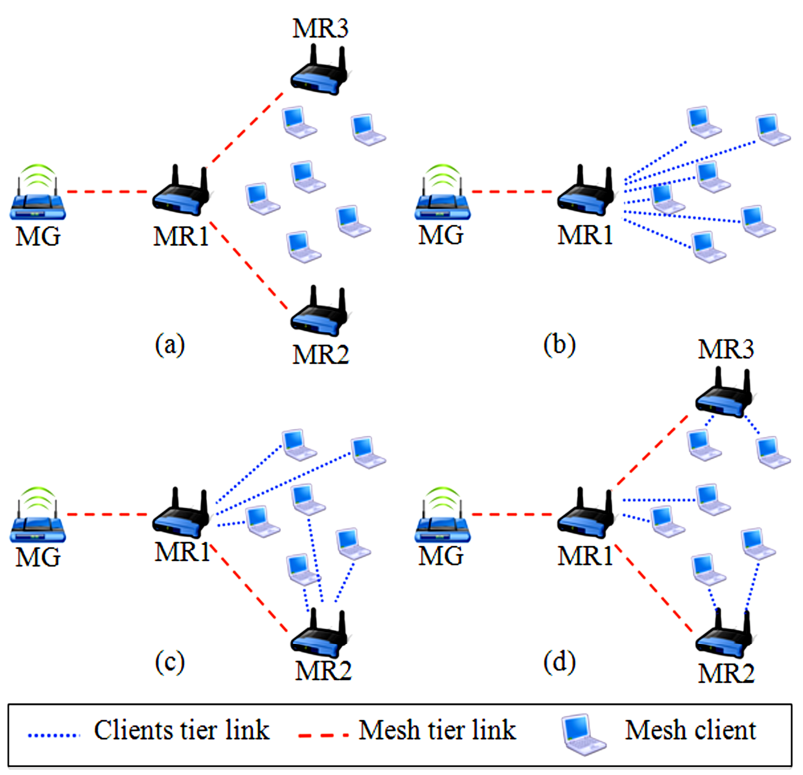

Figure 3. (a) Topology for considered scenario; (b) Subscenario 1, consisting of one active MR; (c) Sub-scenario 2, consisting of two active MRs; (d) Sub-scenario 3, consisting of three active MRs. 
1) Only MR1 is active and all MCs are associated with MR1 as illustrated in Figure 3(b).

2) Either MR1 and MR2, or MR1 and MR3 are active. In this sub-scenario, each MR will have to serve three MCs as illustrated in Figure 3(c).

3) All MRs are active and each has to serve two MCs as illustrated in Figure 3(d).

It is possible to use the concept of BCD to estimate the maximum throughput in both the mesh tier and the clients tier in each of these sub-scenarios so that we can see the effect of number of MRs in this scenario.

\subsection{Sub-scenario 1}

In the clients tier, MR1 serves all six MCs as wireless AP. Since all devices used only one channel within a given cell, we can easily concluded that the $\mathrm{CD}$ of each link is the same set that contain all links; thus all links are BCD (all links need to transmitted same amount of traffic.)By using Equation (3) whereCis the MAC layer capacity of the clients tier or $C_{c}$ and $A M T$ equals to 6, because there are six $\mathrm{MC}$ each offering $1 \mathrm{G}$ traffic.We can concluded that $G_{\max }$ (throughput available to each MC) of clients tier or $C_{c}$ is

$$
G_{c}=\frac{C_{c}}{6}
$$

In the mesh tier, there is only MR1 and the MG. However, the performance should be calculated based on throughput available to each $\mathrm{MC}$ rather than throughput available to each MR. Therefore, the $G_{\max }$ of mesh tiershould be divided by number of MCs served by each MR or 6 in this case to formulate $G_{m}$ of

$$
G_{m}=\frac{C_{m}}{6}
$$

where $G_{m}$ the MAC layer capacity of mesh tier.

\subsection{Sub-scenario 2}

In this sub-scenario, each MR has toserve three MC. Therefore, using Equation (3) and $A M T$ equal to 3 results in $C_{c}$ of

$$
G_{c}=\frac{C_{c}}{3}
$$

However, in the mesh tier there are two operating MR. We can clearly see from Figure 3(c) that $C D$ of both links in the sub-scenario are $\mathrm{BCN}$ and each $\mathrm{CD}$ has to carry $1 G+2 G=3 G$ amount of traffic. By using Equation (3) and $A M T$ equals to 3 and the fact that each MR serves three MCs, the resulting $G_{m}$ is

$$
G_{m}=\frac{C_{m}}{9}
$$

\subsection{Sub-scenario 3}

The clients tier of this sub-scenario has three total active APs and each has to serve two MCs. Using similar analysis to sub-scenario 1 and sub-scenario 2 in previous sections, we can see that $A M T$ is 2 thus resulting with

$$
G_{c}=\frac{C_{c}}{2}
$$

In the mesh tier, similar to sub-scenario 2, we can see from Figure 3(d) that $\mathrm{CD}$ of any link includes all links, and thus are BCNs. In this sub-scenario any BCNs has to carry $1 G+1 G+3 G=5 G(A M T=5)$ amount of traffic and that each MR serves $2 \mathrm{MCs}$, hence, resulting with

$$
G_{m}=\frac{C_{m}}{10}
$$

\subsection{Scenario Discussion}

Since our project is a multiple tiers WMN, the performance of the project will be restricted by the tier with lower performance. We can clearly see from Table 1 or Figure 4 that the performance in the mesh tier decreases with increasing number of MR. This is because of the interference modeled by $\mathrm{BCD}$ concepts. On the other hand, the performance in the clients tier benefits from the extra capacity of additional MR operating in nonoverlapping channel. Therefore, it is important to optimize this trade off, which results from the number of activating MRsin the area, in order to provide the best maximum throughput to each MC.

All metrics are summarized in Table 1 whileFigure 4 shows plots of $G_{m}$ and $C_{c}$ with different value of $G_{m}$ for different $802.11 \mathrm{a}$ data rates $(\mathrm{Gm}-54$ for $54 \mathrm{Mbps}$, $\mathrm{Gm}-24$ for $24 \mathrm{Mbp}$, and $\mathrm{Gm}-12$ for $12 \mathrm{Mbps}$ ). Each value of Cfor both $C_{c}$ and $G_{m}$ are experimentally determined by simulations for each data rate. According to Figure 4, the maximum throughput available to each MC is bounded by either $G_{m}$ or $C_{c}$. Since $G_{m}$ and $C_{c}$ rely on each other, the lower value of the two will be the upper bound of the maximum throughput. Therefore, by using this concept, it is possible to estimate the optimum number of MRs that will give the maximum average throughput to each MC. We can easily see from Figure 4

Table 1. Summary of all values of this section.

\begin{tabular}{ccc}
\hline \multirow{2}{*}{ Sub-scenario } & Metrics \\
\cline { 2 - 3 } 2 & $G_{m}$ & $G_{c}$ \\
\hline 1 & $\frac{C_{m}}{6}$ & $\frac{C_{c}}{6}$ \\
3 & $\frac{C_{m}}{9}$ & $\frac{C_{c}}{3}$ \\
\hline
\end{tabular}




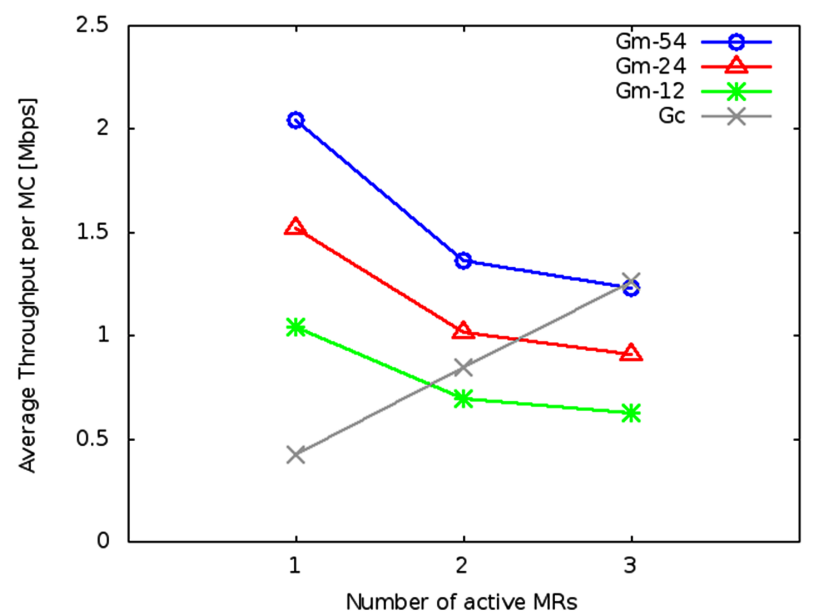

Figure 4. Theoretical value of $G_{m}$ and $G_{c}$ for different data rate.

that the optimum value of MR used for 802.11a with $54 \mathrm{Mbps}$ data rate is three MRs. This is because the capacity in the mesh tier is large enough to accommodate the extra capacity in the clients tier, which results from having three APs operate in nonoverlapping channel.

\section{Simulation Results and Discussion}

Simulations are conducted to confirm this effect discussed in section 3. The simulation scenarios are set up in Qualnet 5.1 with topologies similar to that of Figure 3(b), (c) and (d). Each MR is set up to operate as AP in different nonoverlappingchannel in the clients tier;thus, interference between each MR will not exist within the clients tier. In additional to clients tier interface, each MR also has another interface for communicating in the mesh tier. In mesh tier, all MRs operate in one common channel, which means the collision domains contain all links. Each MC sends CBR traffic to MG and thus all traffic only originate from MCs and flow to MG.

Figure 5 shows results of the simulation scenarios where the line graphs are the theoretical results from Figure 4,and the bar graphs show the simulation results of each mesh tier's data rate (54 Mbps in blue, $24 \mathrm{Mbps}$ in redand $12 \mathrm{Mbps}$ in green.) In the single MR scenario, the simulated results represent the fact that the average throughputs are bounded by $C_{c}$, which has lower value than all $G_{m}$ of all data rate. The results of two operating MRs scenario differ from that of the single operating MR scenario in that the average throughput per MC of $12 \mathrm{Mbps}$ case should now be bounded by $G_{m}$ with data rate of $12 \mathrm{Mbps}$ (in green).This is because the result of $G_{m}$ with data rate of $12 \mathrm{Mbps}$ is now lower than that of $C_{c}$ as shown in Figure 5. However, since the average throughput per $\mathrm{MC}$ of $C_{c}$ is still lower than those of $G_{m}$ of $24 \mathrm{Mbps}$ and $54 \mathrm{Mbps}$, the throughput per MC of those two cases are still bounded by $C_{c}$. Finally, in the

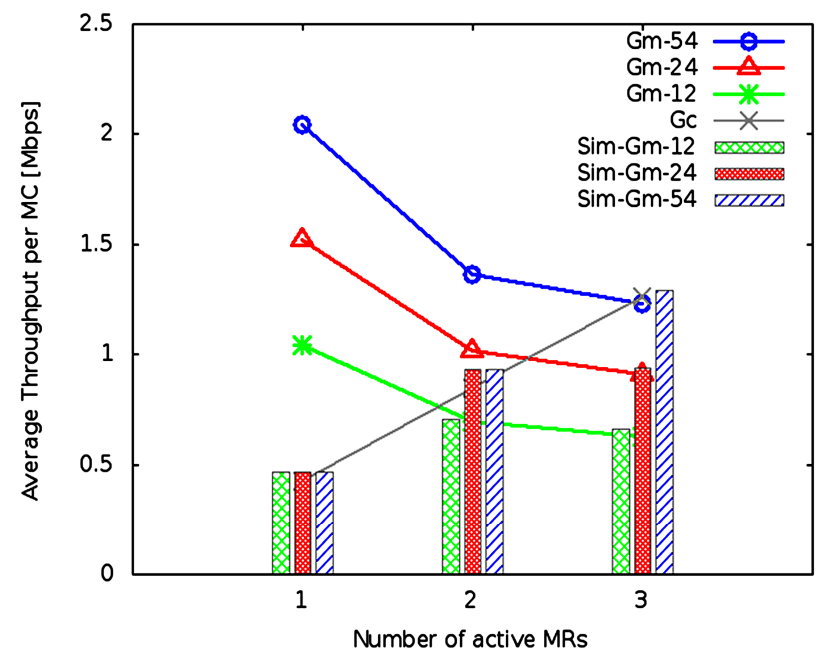

Figure 5. Simulation results and theoretical results for different data rate.

case of 3 active MRs, the theoretical results show that the simulation results should be bounded by $G_{m}$ of each case. As shown in Figure 5, the simulation results clearly follow those of the theoretical results. This shows that the theoretical results are accurate, and we can concluded that we can determine the number of active MR(s), which would yield optimum number of MRs for each $G_{m}$ (of different data rate). From Figure 5, we can deduct that the most optimized number of MRs in $G_{m}$ equals to $54 \mathrm{Mbps}$, $24 \mathrm{Mbps}$, and $12 \mathrm{Mbps}$ are $3 \mathrm{MRs}, 2$ or 3 MRs, and 2 MR respectively. This is because these combinations give the best per MC performance.

\section{Conclusion}

Previous sections show that in order for the project to achieves the optimum performance, the set of active MRs will need to be determined to give the best possible performance. In the future, we aim to apply this idea into a more general case of multiple tiers WMN. In addition, many other important factors such as channel assignment in the mesh tier, and inter-cell interference present in [5] should also be considered when deciding optimum set of active MRs.

In this paper, we briefly explain the national project "The R\&D on the reconfigurable communication resource unit for disaster recovery," which is a unique multiple tiers WMN. We point out that by optimizing the number of active MRs, we can achieve the optimum performanceby using the concept of $\mathrm{BCD}$ and the unique characteristic of multiple tiers WMN. In addition, we also show both theoretical and simulation performance based on a specific scenario of multiple tiers WMN. Our results showed that by choosing the right number or combination of active MRs, we can optimize the performance of the project. 


\section{Acknowledgements}

This work was conducted under the national project, "The R\&D on the reconfigurable communication resource unit for disaster recovery", supported by the Ministry of Internal Affairs and Communications (MIC), Japan.

\section{REFERENCES}

[1] B.S. Manoj and Alexandra Hubenko Baker, "Communication challenges in emergency response," Communications of the ACM - Emergency response information systems: emerging trends and technologies, Vol. 50, No. 3, 2007, pp. 51-53. doi:10.1145/1226736.1226765

[2] P. H. Pathak and R. Dutta, "A Survey of Network Design Problems and Joint Design Approaches in Wireless Mesh Networks," IEEE Communications Surveys \& Tutorials, Vol. 13, No. 3, 2010, pp. 396-428. doi:10.1109/SURV.2011.060710.00062

[3] B. Braunstein, T. Trimble, R. Mishra, B. S. Manoj, L. Lenert and R. R. Rao, "Challenges in Using of Distributed Wireless Mesh Networks,"Proceedings of the $3^{\text {rd }}$ International ISCRAM Conference, Newark, NJ, May 2006

[4] W. Fu and D. P. Agrawal, "Capacity of Hybrid Wireless Mesh Network with Random APs,"IEEE Transactions on Mobile Computing, Vol. 10, No. 12, 2011. doi:10.1109/TMC.2011.247

[5] M. A.Ergin, K. Ramachandran and M.Gruteser, "Understanding the effect of access point density on wireless
LAN performance,"Proceedings of the $13^{\text {th }}$ annual ACM international conference on Mobile computing and networking MobiCom, Montreal, 9-14 September 2007, pp. 350-353. doi:10.1145/1287853.1287902

[6] W. Liu, H. Nishiyama, N. Kato, Y. Shimizu and T. Kumagai, "A Novel Gateway Selection Method to Maximize the System Throughput of Wireless Mesh Network Deployed in Disaster Areas," $23^{\text {rd }}$ IEEE International Symposium on Personal, Indoor and Mobile Radio Communication PIMRC, Sydney, 9-12 September 2012.

[7] J. Bicket, D. Aguayo, S. Biswas and R. Morris, "Architecture and evaluation of an unplanned $802.11 \mathrm{~b}$ mesh network,"MobiCom Proceeding of the $11^{\text {th }}$ annual international conference on Mobile computing and networking, Cologne, August 28- September 2 2005. pp. 31-42. doi: $10.1145 / 1080829.1080833$

[8] J. Camp, J. Robinson, C. Steger and E. Knightly, "Measurement Driven Deployment of a Two-Tier Urban Mesh Access Network,"MobiSys Proceeding of the $4^{\text {th }}$ international conference on Mobile systems, applications and services, Uppsala, 19-22 June 2006. pp. 96-109. doi: $10.1145 / 1134680.1134691$

[9] A. Akella, G. Judd, S. Seshan and P. Steenkiste, "Self-management in chaotic wireless deployments,"MobiCom Proceedings of the $11^{\text {th }}$ annual international conference on Mobile computing and networking, Cologne, August 28 - September 22005.

[10] J. Jun and M. L. Sichitiu, "The Nominal Capacity of Wireless Mesh Networks," IEEE Wireless Communications, Vol. 10, No. 5, 2003, pp. 8-14. doi:10.1109/MWC.2003.1241089 